\title{
Crohn's disease with ankylosing spondylitis in an adolescent patient who had undergone long ileo-colonic anastomosis for Hirschsprung's disease as an infant
}

\author{
Ha Yeon $\mathrm{Kim}^{1 *}$, Tae Wook Kim²* \\ ${ }^{1}$ Department of Internal Medicine, Dong-A University College of Medicine, Busan, ${ }^{2}$ Department of Internal Medicine, \\ Biomedical Research Institute, Pusan National University Hospital, Pusan National University School of Medicine, Busan, Korea
}

Crohn's disease (CD) is a chronic, idiopathic, inflammatory disorder of the gastrointestinal tract. In rare cases, CD has been associated with Hirschsprung's disease (HD); however, the underlying pathophysiology of this and other comorbidities is not yet fully understood. In this report, we describe the case of a 17-year-old patient who was diagnosed with both CD and ankylosing spondylitis (AS), having undergone a long ileo-colonic anastomosis to treat HD at 12 months of age. To our knowledge, this is the first documented case of CD combined with AS in a patient with HD. (Intest Res 2017;15:133-137)

Key Words: Crohn disease; Spondylitis, ankylosing; Hirschsprung disease; Inflammatory bowel diseases; Long ileo-colonic anastomosis

\section{INTRODUCTION}

Hirschsprung's disease (HD) is a motor disorder of the gastrointestinal tract that is caused by a failure of neural crest cell migration during intestinal development. The incidence of HD is approximately one in 5,000 live births, ${ }^{1}$ and the main treatment is surgical resection of the affected segment. Most patients experience normal or near-normal bowel function after definitive surgery for HD. One complication of surgery for HD is enterocolitis, which presents as watery, bloody diarrhea combined with abdominal distension. ${ }^{2}$

$\mathrm{CD}$ is the generic name for a group of multisystem disorders with specific clinicopathological features, including fo-

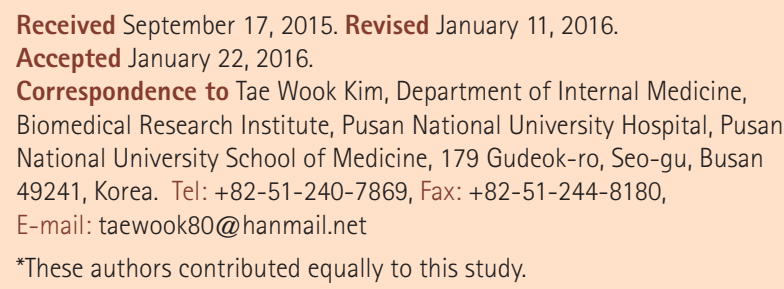

Financial support: None. Conflict of interest: None. cal, asymmetric, transmural, and occasionally granulomatous inflammation primarily affecting the gastrointestinal tract.

Ankylosing spondylitis (AS) is common forms of chronic inflammatory arthritis and the archetypal spondyloarthritis. ${ }^{3}$ The clinical signs of AS include sacroiliitis, inflammatory back pain, morning stiffness, acute anterior uveitis, and IBD. Between $5 \%$ and $10 \%$ of AS cases are associated with IBD and either CD or UC. ${ }^{3}$

A study into the comorbidities of IBD showed that UC alone, and not $\mathrm{CD}$, is associated with $\mathrm{HD} .{ }^{4} \mathrm{In}$ fact, $\mathrm{CD}$ comorbid with HD is extremely rare-to our knowledge, only two cases have been reported in the medical literature to date, and HD combined with both CD and AS has never been described.

In this report, we describe the case of a patient who had undergone surgery for HD as an infant, and was later diagnosed with CD combined with AS.

\section{CASE REPORT}

A 17-year-old boy was referred from a secondary medical center to our gastrointestinal center because of chronic

\footnotetext{
๑ Copyright 2017. Korean Association for the Study of Intestinal Diseases. All rights reserved.

This is an Open Access article distributed under the terms of the Creative Commons Attribution Non-Commercial License (http://creativecommons.org/licenses/by-nc/4.0)

which permits unrestricted non-commercial use, distribution, and reproduction in any medium, provided the original work is properly cited.
} 
abdominal pain and diarrhea that had persisted for several years. Watery diarrhea occurred four times a day and was associated every other day with dull pain in the lower abdomen. He had been diagnosed with HD and undergone ileostomy at the age of 4 months; this was followed by a subtotal colectomy at 12 months of age, where surgeons used the Lester Martin modification of the Duhamel procedure. Since the age of 5 years, he had been repeatedly hospitalized for postoperative enterocolitis; he also had a history of uveitis, and had suffered from visual disturbance in the right eye since the age of 15 years. He had been experiencing recurring aggravation of pain in the left ankle and knee, as well as in the back, until about 3 years prior to his visiting our center. He had no family history of IBD.

The patient was $167 \mathrm{~cm}$ tall and weighed $50 \mathrm{~kg}$ (BMI, 17.92). Upon admission, his vital signs were blood pressure, 110/70 mmHg; pulse rate, 72 beats per minute; respiratory rate, 20 breaths per minute; body temperature, $36.4^{\circ} \mathrm{C}$. He appeared chronically ill, with pale conjunctivae. Abdominal examination revealed increased bowel sounds and tenderness in the lower abdomen, although no rebound tenderness or palpable abdominal masses were found, and rectal examination revealed no perianal disease. Slight edema and tenderness were observed in the left ankle and knee. A routine complete blood count detected hypochromic, microcytic anemia, as well as thrombocytosis-white blood cell count, $6.130 \times 10^{3} / \mu \mathrm{L}$; hemoglobin level, $5.5 \mathrm{~g} / \mathrm{dL}$ (mean corpuscular volume, $54.4 \mathrm{fL}$; mean cell hemoglobin, 21.4 pg); platelet count, $587 \times 10^{3} / \mu \mathrm{L}$. Further laboratory tests revealed the following abnormalities-iron, $30 \mu \mathrm{g} / \mathrm{dL}$; total iron-binding capacity, $394 \mu \mathrm{g} / \mathrm{dL}$; ferritin, $16.12 \mathrm{ng} / \mathrm{mL}$; Creactive protein level, $3.36 \mathrm{mg} / \mathrm{dL}$. No Clostridium difficile toxin A was detected in a stool sample, and stool culture and parasite tests were negative.

Enhanced CT of the abdomen and pelvis revealed a thickened wall in the small bowel (neorectosigmoid junction, neosigmoid colon, and neoascending colon). The proximal parts of the narrowed areas were dilated (Fig. 1). Colonoscopy revealed a normal rectal mucosa, but edematous mucosa and superficial ulcerations were observed above 10 $\mathrm{cm}$ from the anal verge. In fact, we were unable to advance the endoscope beyond $15 \mathrm{~cm}$ from the anal verge, because the intestinal lumen was so narrowed (Fig. 2). A small bowel series revealed narrowing and streaky mucosal changes in the proximal small bowel and at the site of anastomosis, as well as multifocal stricture in the small bowel (especially the neoascending colon and neosigmoid colon) and around the site of anastomosis, with marked luminal dilatation consistent with CD (Fig. 3). On the basis of these endoscopic and imaging findings, as well as his symptoms, the patient was diagnosed with $\mathrm{CD}$.

While the diagnosis was being made, the patient's uveitis worsened, as did his ankle and knee arthritis, and his back pain. Human leukocyte antigen B27 was detected in serum, and MRI of the pelvis revealed sacroiliitis. After consultation with rheumatologists, CD combined with AS was diagnosed.

Subsequently, we injected intravenous steroids and metronidazole; to treat the CD combined with AS, a course of infliximab injection was started. The patient's abdominal pain and diarrhea improved during hospitalization, and he
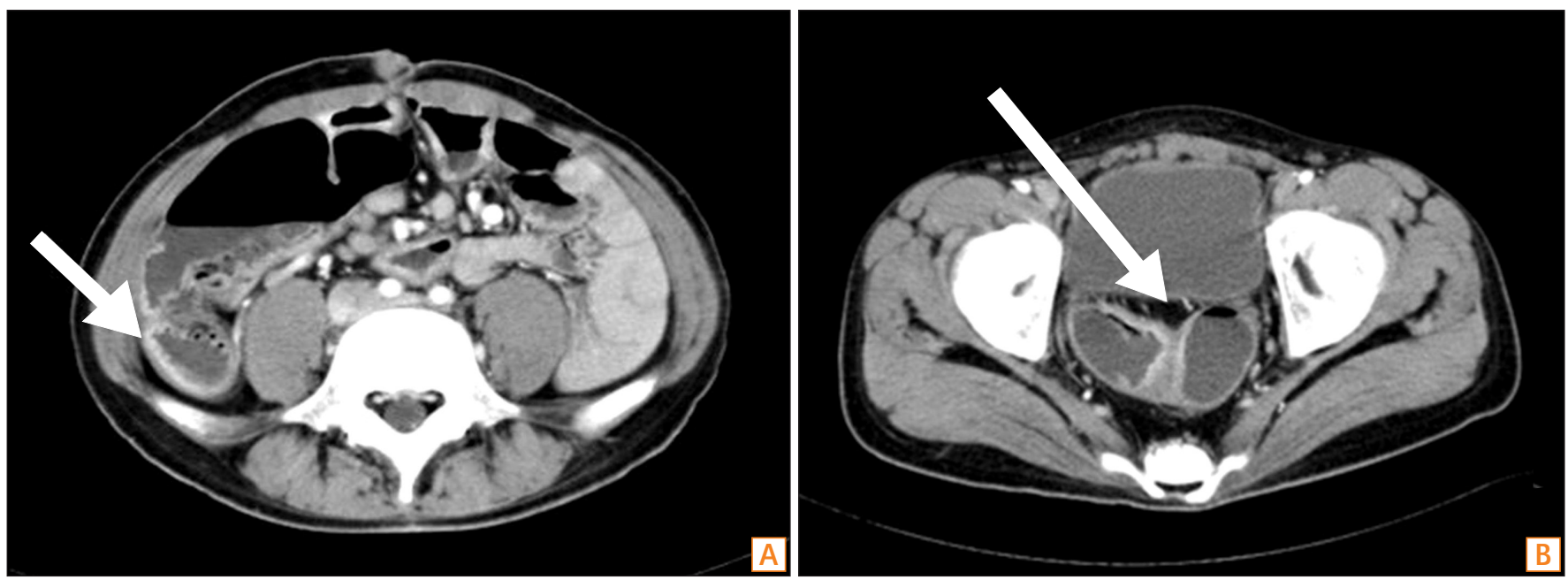

Fig. 1. Abdominal CT findings. Multiple strictures were accompanied by dilation of the small bowel. (A) A thickened mucosal wall was seen at the neoascending colon portion of ileum (arrow). (B) A thickened mucosal wall was also noted at the neosigmoid colon portion of ileum and anastomosis site (arrow). 

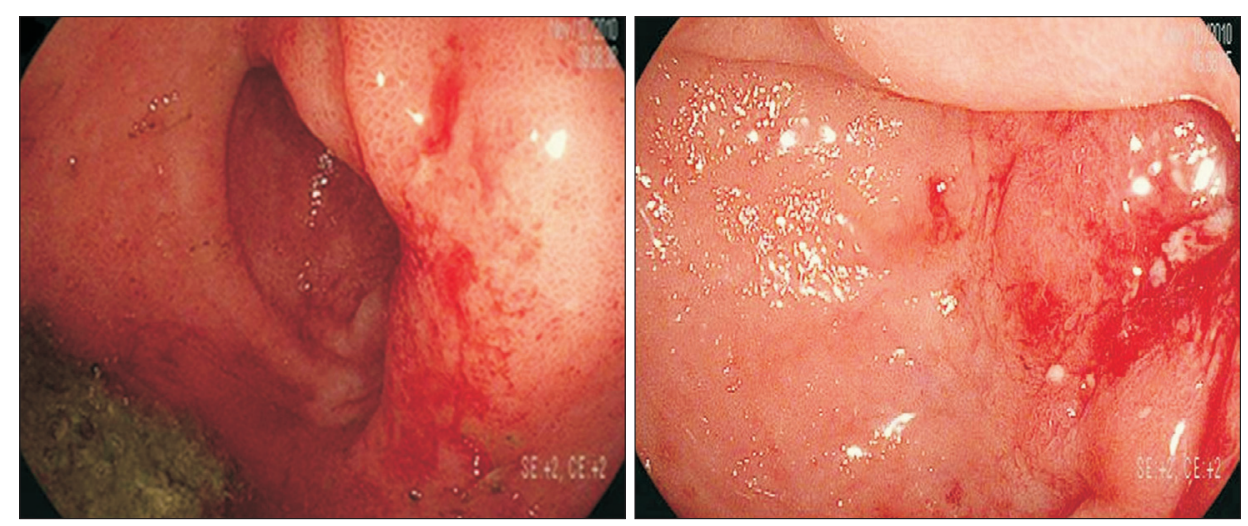

Fig. 2. Colonoscopic findings. Edematous mucosa and a superficial ulcerative lesion were observed above $10 \mathrm{~cm}$ from the anal verge. The scope could not pass through because of luminal narrowing.
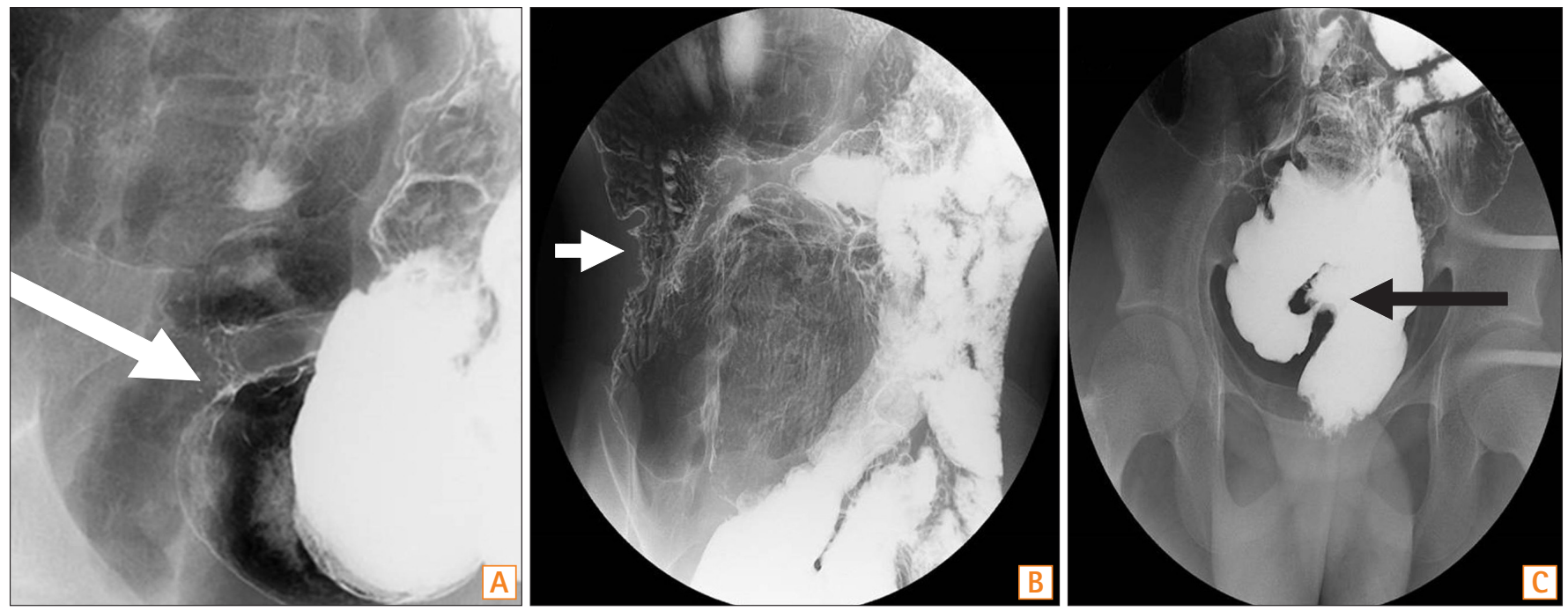

Fig. 3. Small bowel series findings. (A) Luminal narrowing was seen at neoterminal ileum (white arrow). (B) An irregular and thickened wall was observed at neoascending colon portion of ileum (white arrow). (C) A suspected focal stricture was observed in the neosigmoid colon portion of ileum (black arrow).

was discharged on admission day 12. Twelve months after discharge, he was evaluated by follow-up colonoscopy. The mucosal edema and ulcerations that had been observed above $10 \mathrm{~cm}$ from the anal verge had improved, but small bowel evaluation was limited by an acute luminal angle. The patient is currently within a 64-month observation period at Dong-A University Hospital. He has tolerable gastrointestinal symptoms, but is being administered infliximab injections to treat uveitis.

\section{DISCUSSION}

HD is characterized by the congenital absence of ganglion cells in both the submucosal (Meissner's) and myenteric (Auerbach's) plexuses; the disease is caused by a failure of neural crest cell (enteric ganglion cell precursors) migration during intestinal development. The resulting aganglionic segment of the colon is unable to relax, leading to a functional obstruction. Many children with HD improve after surgical treatment; nonetheless, long-term follow-up studies have identified a number of concerns. The most commonly encountered problems include constipation, incontinence, and enterocolitis. Indeed, Hirschsprung-associated enterocolitis is a major cause of postoperative morbidity and occasional mortality. ${ }^{7}$ This complication occurs after the definitive pullthrough procedure at a frequency of $30 \%$ to $40 \%$, usually between 3 weeks and 20 months after surgery. ${ }^{2,8,9}$ Occasionally, Hirschsprung-associated enterocolitis needs to be differentiated from IBD or infectious enterocolitis.

There is no single gold standard for CD diagnosis. The disease is diagnosed on the basis of the clinical findings (detailed history, physical examination, endoscopy, histol- 
ogy, radiology, and laboratory investigations).$^{10}$ However, it remains challenging to differentiate $\mathrm{CD}$ from other disorders such as acute infectious colitis, intestinal tuberculosis, UC, and Behçet's colitis. In particular, the postoperative state of HD mimics that of enterocolitis. Both conditions present with diarrhea, hematochezia, fever, abdominal pain, and distension. However, postoperative HD can be distinguished from postoperative enterocolitis based on the former's recurrent gastrointestinal symptoms, extraintestinal manifestations, radiological findings (small bowel stricture, fistula, etc.), onset time, perianal disease, and therapeutic response to immunosuppression.

The association between AS and human leukocyte antigen B27 is weaker in IBD-related AS than in idiopathic AS, and there is evidence of an association between AS-related gut inflammation and CD-related CARD15 mutations. ${ }^{3}$ Currently, infliximab is the drug of choice to treat patients with active AS associated with IBD. ${ }^{3}$

In our patient, $\mathrm{CD}$ and AS were diagnosed 16 years after HD, although he had repeatedly been hospitalized-up to five or six times a year-for recurrent diarrhea and abdominal pain. At each hospitalization, he had been diagnosed with enterocolitis and treated using antibiotics and supportive care. Subsequently, because of his recurring symptoms, severe anemia, and extraintestinal manifestations (back pain, arthritis, and uveitis) - which had persisted for several years-the patient was transferred to our hospital. He was then diagnosed with CD combined with AS on the basis of endoscopic, laboratory, radiographic, and MRI findings. Thus, in patients who have undergone surgery to treat HD, and who present with recurrent diarrhea, abdominal pain with anemia, and extraintestinal manifestations, CD should be considered. In so doing, clinicians will avoid delayed CD diagnosis.

The immunological features common to HD and CD include increased expression of the major histocompatibility complex II and intercellular adhesion molecule I antigens. ${ }^{11-13}$ Although each disease is associated with specific chromosomal disorders, none of these is common to both conditions. ${ }^{14,15}$ Moreover, no association between HD and $\mathrm{CD}$ has been previously reported. However, we would suggest that our patient has a genetic mutation that makes him susceptible to CD and contributes to infections by multiple pathogens (e.g., Salmonella spp., Shigella spp., Campylobacter spp., and C. difficile) that are more frequent than in patients who have not undergone surgery. These recurrent infections may have induced CD by triggering an inflammatory response that could not be controlled by the mucosal immune system. Further study on the association between $\mathrm{CD}$ and HD can provide a better understanding of the pathophysiology of CD.

In summary, CD occurs very rarely after surgery for HD, and diagnosis of the condition is challenging. Although postoperative enterocolitis is more common in patients with HD, CD should be considered in those who (1) present with multiple episodes of enterocolitis over a long time period (1-2 years) after a definitive pull-through procedure and (2) have atypical symptoms such as arthritis, uveitis, and erythema nodosum.

\section{REFERENCES}

1. Okamoto E, Ueda T. Embryogenesis of intramural ganglia of the gut and its relation to Hirschsprung's disease. J Pediatr Surg 1967;2:437-443.

2. Engum SA, Grosfeld JL. Long-term results of treatment of Hirschsprung's disease. Semin Pediatr Surg 2004;13:273-285.

3. Rudwaleit M, Baeten D. Ankylosing spondylitis and bowel disease. Best Pract Res Clin Rheumatol 2006;20:451-471.

4. Cucino C, Sonnenberg A. The comorbid occurrence of other diagnoses in patients with ulcerative colitis and Crohn's disease. Am J Gastroenterol 2001;96:2107-2112.

5. Kessler BH, So HB, Becker JM. Crohn's disease mimicking enterocolitis in a patient with an endorectal pull-through for Hirschsprung's disease. J Pediatr Gastroenterol Nutr 1999;29: 601-603.

6. Dray X, Sokol H, Vahedi K, Lavergne-Slove A, Marteau P. Pure ileal Crohn's disease without colonic involvement after a long ileo-colonic anastomosis (Lester Martin Procedure) for Hirschsprung's disease: an argument favoring a specific sensitivity of the ileum in a subset of patients with Crohn's disease. Inflamm Bowel Dis 2007;13:243-244.

7. Imamura A, Puri P, O'Briain DS, Reen DJ. Mucosal immune defence mechanisms in enterocolitis complicating Hirschsprung's disease. Gut 1992;33:801-806.

8. Hackam DJ, Filler RM, Pearl RH. Enterocolitis after the surgical treatment of Hirschsprung's disease: risk factors and financial impact. J Pediatr Surg 1998;33:830-833.

9. Kleinhaus S, Boley SJ, Sheran M, Sieber WK. Hirschsprung's disease: a survey of the members of the Surgical Section of the American Academy of Pediatrics. J Pediatr Surg 1979;14:588-597.

10. Ye BD, Jang BI, Jeen YT, et al. Diagnostic guideline of Crohn's disease. Korean J Gastroenterol 2009;53:161-176. 
11. Hirobe S, Doody DP, Ryan DP, Kim SH, Donahoe PK. Ectopic class II major histocompatibility antigens in Hirschsprung's disease and neuronal intestinal dysplasia. J Pediatr Surg 1992;27:357-362.

12. Kobayashi H, Hirakawa H, Puri P. Overexpression of intercellular adhesion molecule-1 (ICAM-1) and MHC class II antigen on hypertrophic nerve trunks suggests an immunopathologic response in Hirschsprung's disease. J Pediatr Surg 1995;30:16801683.
13. Morise K, Yamaguchi T, Kuroiwa A, et al. Expression of adhesion molecules and HLA-DR by macrophages and dendritic cells in aphthoid lesions of Crohn's disease: an immunocytochemical study. J Gastroenterol 1994;29:257-264.

14. Lyonnet S, Bolino A, Pelet A, et al. A gene for Hirschsprung disease maps to the proximal long arm of chromosome 10. Nat Genet 1993;4:346-350.

15. Curran ME, Lau KF, Hampe J, et al. Genetic analysis of inflammatory bowel disease in a large European cohort supports linkage to chromosomes 12 and 16. Gastroenterology 1998;115:1066-1071. 